Portland State University

PDXScholar

\title{
BRET Assays to Determine Altered Function of a D2DR Variant in G Protein-Independent and -Dependent Pathways
}

Alex R. Kunz

Portland State University

Follow this and additional works at: https://pdxscholar.library.pdx.edu/honorstheses

Let us know how access to this document benefits you.

\section{Recommended Citation}

Kunz, Alex R., "BRET Assays to Determine Altered Function of a D2DR Variant in G Protein-Independent and -Dependent Pathways" (2019). University Honors Theses. Paper 785.

https://doi.org/10.15760/honors.803

This Thesis is brought to you for free and open access. It has been accepted for inclusion in University Honors Theses by an authorized administrator of PDXScholar. Please contact us if we can make this document more accessible: pdxscholar@pdx.edu. 
BRET Assays to Determine Altered Function of a $D 2 D R$ Variant in G proteinIndependent and -Dependent Pathways

\author{
By Alex Kunz
}

An undergraduate honors thesis submitted in partial fulfillment of the requirements for the degree of Bachelor of Science in

University Honors

and

Biochemistry

Thesis Adviser

Dr. Kim Neve 


\begin{abstract}
:
$\mathrm{G}$ protein-dependent and independent signaling pathways perform crucial roles in dopaminerelated physiological and pathological processes. A pivotal role of dopamine in the CNS is the control of locomotion; disruption to dopaminergic systems, such as reduced striatal D2 receptor availability, contributes to the development of movement disorders. Affected members of a family with dystonia, a movement disorder, are heterozygous for a SNP in exon 4 of D2DR (c.634A>T p.I212F), a missense variant located within the third intracellular loop; specifically, the variation is in the middle of a short stretch of residues that have been found to be critical for binding of arrestin and several other dopamine receptor-interacting proteins. The comparative actions between D2-WT and D2-I212F were analyzed in their effects on both the G proteinmediated pathway and the arrestin3 pathway. It was shown that the D2-I212F variant was only $34 \%$ as effective as D2-WT at recruiting arrestin3, a deficiency that is likely to have important behavioral consequences. Additionally, quinpirole stimulation of D2-I212F produced dosedependent inhibition of cAMP accumulation; it was observed that lower concentrations of quinpirole were able to fully activate the rare variant. To further investigate whether this missense variant causes movement disorders, a condition that mimics the heterozygosity (D2WT/D2-I212F), for the SNP affected patients have, was utilized and tested. Co-transfection of D2-WT and D2-I212F trended toward a shift in the EC50, where D2-WT/D2-I212F were different from D2-WT expressed alone, but the difference was not statistically different. This variant is likely to have decreased arrestin-mediated signaling and enhanced G protein-mediated signaling, so behaviors and cellular functions mediated by those two pathways will be altered according to patients with this variant.
\end{abstract}




\begin{abstract}
Abbreviations:
adenylyl cyclase (AC) - bioluminescence resonance energy transfer (BRET) - cyclic adenosine monophosphate (cAMP) - CAMYEL (cAMP sensor using YFP-Epac-Rluc) - DMEM (Dulbecco's modified Eagle's medium ) - dopamine (DA) - D2 dopamine receptor (D2DR) D2L (D2- long) - D2S (D2- short) - Epac (exchange protein directly activated by cAMP) - FBS (fetal bovine serum) - green fluorescent protein (GFP) - GRK (G protein coupled receptor kinase) - G protein coupled receptors (GPCR) - HD (Huntington's Disease) - HEK293 (Human embryonic kidney 293) - PBS (phosphate buffered saline) - pDNA (plasmid deoxyribonucleic acid) - PEI (polyethyleneimine) - Renilla luciferase (Rluc) - SNP (single-nucleotide polymorphism) - third intracellular loop (IL3) - transmembrane (TM) - yellow fluorescent protein (YFP)
\end{abstract}

\title{
Introduction:
}

\section{Dopamine Receptors}

Dopamine (DA) receptors are members of the family of seven-transmembrane (7-TM) proteins that interact with distinct signaling molecules on the extracellular domain, inducing subsequent changes in the structure of the intercellular domain. 7-TM proteins are the largest family of transmembrane protein receptors, most of them signal via heterotrimeric $\mathrm{G}$ proteins and are therefore called G protein-coupled receptors (GPCRs) (Liu et al., 2007; Pierce and Lefkowitz, 2001; Neve, 2010). GPCRs are categorized into three groups, in which dopamine receptors are classified within the largest subgroup Class A (Neve, 2010). This subgroup is comprised of receptors that respond to biogenic amines, peptides, and odorants. DA receptors respond when bound by their endogenous ligand dopamine, a biogenic amine.

\subsection{Dopamine Receptor Types and Gene Organization}

There are two broad classes of DA receptors, D1- and D2- like DA receptors (Neve, 2010; Usiello et al., 2000). Gene organization for either can be divided up into coding and regulatory regions; moreover, D1-like receptor genes contain only one exon and no introns, whereas D2like receptor genes contain several coding exons positioned in-between introns. The D1subfamily comprises the D1 and D5 DA receptors and the D2-subfamily comprises the D2, D3, 
and D4 DA receptors. D2 dopamine receptors (D2DR) are encoded by genes that contain introns and are further divided into respective isoforms (Neve, 2010; Usiello et al., 2000). The alternative splicing of an 87-bp exon in the D2 receptor gene results in two transcript variants that encode the distinct isoforms D2-short (D2S) and D2-long (D2L) (Usiello et al., 2000). D2L has 29 more amino acid residues, in the third intracellular loop (IL3) between TM domains 6-7, than D2S as the result of the alternatively spliced exon that is present in the former but spliced out of the latter (Usiello et al., 2000). IL3 is involved in the coupling of D2DR to different G proteins.

$\mathrm{G}$ proteins are guanine nucleotide binding proteins that bind to and hydrolyze guanosine triphosphate (GTP) to guanosine diphosphate (GDP). The heterotrimeric G protein complexes are composed of three subunits: alpha, beta, and gamma (Hilger et al., 2018; Tuteja 2009). D1and D2-like receptors couple to different heterotrimeric G-proteins; D1-like receptors signal via $\mathrm{G} \alpha_{\mathrm{s}}$ and $\mathrm{G} \alpha_{\text {olf }}$ whereas D2-like receptors signal via $\mathrm{G} \alpha_{\mathrm{i}}$ and $\mathrm{G} \alpha_{\mathrm{o}}$, each activating a unique set of signaling pathways (Hilger et al., 2018; Tuteja 2009).

\subsection{G protein-Coupled Receptor Signaling Pathways}

D2DR is a GPCR that undergoes a conformational change upon activation by a ligand, initiating subsequent signaling through $\mathrm{G}$ proteins as well as through $\mathrm{G}$ protein-independent pathways. The former activity is due to the exchange of GDP/ GTP associated with the G $\alpha$ subunit, a function of D2DR as a guanine nucleotide exchange factor (Tuteja 2009). Upon the exchange, the $G \beta / G \gamma$ dimer dissociates from $G \alpha$, allowing each moiety to act upon their downstream effectors. One effect of G protein activation by D2DR is the inhibition of adenylyl cyclase (AC) activity, which decreases the production of cyclic adenosine monophosphate 
(cAMP), a second messenger used for intracellular signal transduction in many biological processes. Following signal propagation, the GTP associated with the G $\alpha$ subunit is hydrolyzed to GDP, deactivating and subsequently re-associating G $\alpha$ with the $G \beta / G \gamma$ dimer, forming the inactive heterotrimeric complex (Tuteja 2009). Signal responses can also be attenuated by the phosphorylation of D2DR by G protein coupled receptor kinases (GRKs), allowing for the propagation of alternative signaling pathways (Beaulieu et al., 2005).

Upon activation of D2DR, signaling can be directed toward heterotrimeric G proteinindependent pathways by means of arrestin 3 recruitment. The activated receptor is a substrate for GRK 2/3, which phosphorylates serine and threonine residues in IL3 of D2DR. A segment of residues, 212-215, in IL3 of D2DR is also required for recruitment of arrestin to the receptor and receptor internalization (Lan et al., 2009). In arrestin-dependent receptor internalization, arrestins act as adapters for $\beta(2)$ adaptin and clathrin by bringing activated receptors to clathrin-coated pits for endocytosis (DeWire et al., 2007). In addition to the critical roles of arrestin in receptor internalization and termination of $\mathrm{G}$ protein-dependent signaling, arrestins function as scaffolds for signaling proteins. In one such case, a signaling complex is formed in which arrestin3 mediates the interaction of protein kinase $\mathrm{B}(\mathrm{Akt})$ and protein phosphatase $2 \mathrm{~A}$ in response to agonist activation of D2DR (Beaulieu et al., 2005). This signaling mechanism may be involved in the expression of locomotive responses to dopaminergic drugs, such as amphetamine and apomorphine (Beaulieu et al., 2005).

\section{Bioluminescence Resonance Energy Transfer (BRET)}

Bioluminescence resonance energy transfer (BRET) is a transfer of energy between a donor enzyme and a fluorophore (acceptor), upon being in close proximity (10-100 ̊ apart), 
whereby the enzyme transfers its excited-state energy to the fluorophore, a ground-state acceptor, resulting in light emission at a longer wavelength. In nature when the photoprotein aequorin transfers energy to green fluorescent protein (GFP), it emits green light as seen in the jellyfish, Aequorea victoria; in vitro, purified aequorin emits blue light, a shorter wavelength, in the absence of GFP (Xu et al., 1999). This naturally occurring process can be used in the laboratory to measure protein interactions in vivo and in vitro. Since the first use of BRET by Xu et al. (1999) the technique has been used to study protein-protein interactions well over a thousand times, half of which involve GPCR interactions (Pfleger et al., 2006).

\subsection{BRET Assay to Study Arrestin Recruitment}

Ligand induced recruitment of arrestin by GPCRs, has been dynamically monitored using BRET (Donthamsetti et al., 2015; Pfleger et al., 2006; Stoddart et al., 2015). To study arrestin recruitment by GPCRs, proteins are modified by means of fusion to accessory protein fragments, that act as donor and acceptor. In many of these studies, the GPCRs are fused to a BRET donor that are variants of the enzyme Renilla reniformis luciferase (Rluc), whereas the other interacting proteins are fused to fluorescent acceptors that are variants of GFP. In our case, D2DR was fused to Rluc8, a variant of the luciferase protein (Clayton et al., 2014) and arrestin3 was coupled to a yellow fluorescent protein (YFP) called mVenus. The oxidation of the substrate, coelenterazine $h$, upon enzymatic cleavage by Rluc8 produces luminescence that excites the fluorophore mVenus, resulting in fluorescence emittance. The intensity of light emissions by Rluc8 and mVenus are measured and the ratio is calculated as the BRET signal. The intensity of the BRET signal will vary based on the relative distance between Rluc 8 and mVenus, indicating arrestin 3 recruitment to D2DR.

\subsection{CAMYEL Assay to Study cAMP Regulation}


Ligand induced cAMP regulation by GPCRs has been dynamically monitored using a BRET sensor for cAMP, CAMYEL (cAMP sensor using YFP-Epac-Rluc) (Jiang et al., 2007; Matthiesen and Nielsen, 2011). CAMYEL is composed of a cAMP binding protein, Epac (exchange protein directly activated by $\underline{\mathrm{c}} \mathrm{AMP}$ ), that contains a cAMP binding domain that serves as a molecular switch for detecting intracellular second messenger cAMP levels (Valkovic et al., 2018). Additionally, Epac in CAMYEL is flanked by a BRET pair: variants of Rluc and GFP.

When cAMP is absent, Epac assumes a closed conformation such that the energy donor Rluc and energy acceptor GFP are in close proximity, producing a higher BRET signal; conversely, Epac assumes an open conformation upon binding by cAMP allowing Rluc and GFP to move further apart, resulting in a loss of BRET (Salahpour, 2012). In this study, the inhibition of cAMP accumulation, by the agonist quinpirole, was measured using CAMYEL.

\section{Problem and Objective:}

Dopamine plays a critical role in the way our brain controls our movements. Disruption to dopaminergic systems is known to play a role in movement disorders. The foremost dopaminergic system concerned in motor control arises from the substantia nigra (SN). Progressive degeneration of dopaminergic neurons in the nigrostriatal system and subsequent loss of dopamine in the striatum are causes of Parkinson's disease, a neurodegenerative disorder affecting the motor system (Lizarraga et al., 2016). Huntington's Disease (HD), a neurodegenerative disorder, is caused by a CAG trinucleotide repeat expansion in the HTT gene. HD is characterized by involuntary movement and psychological symptoms, in which modifications in dopamine function and neurotransmission play a significant role (Cepeda et al., 2014). The progression of HD is accompanied by reduction in striatal D1- and D2- receptors; this 
type of reduction is often associated with movement disorders (Lizarraga et al., 2016). It is conceivable that $\mathrm{D} 2$ receptor dysfunction may lead to similar movement disorders.

A neurological disorder with autosomal dominant transmission, that produces impaired movement similar to HD, has been identified within a Dutch pedigree. The single-nucleotide polymorphism (SNP) associated with this disorder occurs in the coding region of the $D 2 D R$ gene, which encodes the D2 subtype of the dopamine receptor. The missense variant in D2DR (c.634A>T p.I212F) is within the IL3 in the middle of a short stretch of residues that have been found to be critical for binding of arrestin and several other dopamine receptor-interacting proteins, as well as for normal expression and agonist-induced internalization of the D2 receptor. Preliminary data from the Neve lab indicate that mutating D2 receptor Ile ${ }^{212}$ to Phe decreases the recruitment of arrestin to the receptor. The major objective of this project is to test the hypothesis that the D2DR c.634A>T variant $\left(\mathrm{Phe}^{212}\right)$ causes movement abnormalities by showing that the variant has altered function.

\section{Materials and Methods:}

Human embryonic kidney 293 (HEK293) cells were cultured in Dulbecco's modified Eagle's medium (DMEM), supplemented with 10\% fetal bovine serum (FBS; Thermo Fisher Scientific, Waltham, MA) at $37^{\circ} \mathrm{C}$ with $5 \% \mathrm{CO} 2$.

\section{BRET}

HEK293 cells were co-transfected with plasmids, based on the assay (arrestin3 recruitment or CAMYEL). Constructs were transiently transfected into HEK293 cells using polyethyleneimine (PEI; Polysciences, Inc., Warrington, Pa) at a ratio of $3 \mu \mathrm{L}$ of PEI ( $1 \mu \mathrm{g} / \mu \mathrm{L})$ per $1 \mu \mathrm{g}$ of plasmid DNA (pDNA) for six hours, after which the medium was replaced with fresh 
DMEM supplemented with 10\% FBS. Forty-eight hours after transfection, cells were treated with a trypsin-EDTA solution for disengagement and subsequent harvest from culture plates. Cells were then resuspended in BRET assay buffer (PBS with $0.1 \mathrm{mM} \mathrm{CaCl} 2,0.5 \mathrm{mM} \mathrm{MgCl} 2$ and $0.2 \mu \mathrm{M}$ ascorbic acid) and plated at $(\sim 100,000$ cells in $80 \mu \mathrm{L}) /$ well in 96 -well OptiPlates (PerkinElmer Life Sciences, Waltman, MA). Plates were incubated at $37^{\circ} \mathrm{C}$ with $5 \% \mathrm{CO} 2$ for 1 hour, followed by the addition of $10 \mu \mathrm{L}$ of agonist, at concentrations ranging between $0.1 \mathrm{nM}$ and $10 \mu \mathrm{M}$, or vehicle. Additionally, $10 \mu \mathrm{L}$ of coelenterazine $h$ was added giving a final concentration of $2 \mu \mathrm{M}$. Emission of the donor and acceptor was measured ten minutes after the addition of the agonist using a Victor ${ }^{\mathrm{TM}} \mathrm{X}$ Light luminescence reader (PerkinElmer Life Sciences, Waltman, MA).

\subsection{Arrestin3 Recruitment Assay}

HEK293 cells were co-transfected with plasmids containing GRK2 (Clayton et al., 2014.), the receptors D2-WT or D2-I212F fused to Rluc8 (constructed in the lab), and arrestin3 fused to mVenus to measure D2DR-mediated arrestin3 recruitment. The agonist added was dopamine. The BRET signal was calculated according to the following equation:

$$
\frac{\text { Donor }+ \text { Acceptor emission at } 530 \mathrm{~nm}}{\text { Donor }+ \text { Acceptor emission at } 460 \mathrm{~nm}}-\frac{\text { Donor emission at } 530 \mathrm{~nm}}{\text { Donor emission at } 460 \mathrm{~nm}}
$$

Data were analyzed by nonlinear regression using log[agonist] vs. response(three parameters) in Prism (GraphPad, San Diego, CA). The basal response was subtracted from the maximal dopamine stimulation response for the maximal arrestin3 recruitment analysis.

\subsection{CAMYEL Assay}


HEK293 cells were co-transfected with plasmids containing the receptors D2-WT or D2I212F, and a CAMYEL sensor (Jiang et al., 2007) to measure D2DR-mediated inhibition of cAMP. The agonist added was quinpirole. Additionally, $10 \mu \mathrm{L}$ of forskolin was added to give a final concentration of $10 \mu \mathrm{M}$. The BRET ratio was calculated by dividing emission at $460 \mathrm{~nm}$ by the emission at $535 \mathrm{~nm}$. This assay was performed four times, with measurements made by quadruplicate or triplicate. Data were analyzed by nonlinear regression using log[inhibitor] vs. response(three parameters) and $\log$ (inhibitor) vs. normalized response in Prism (GraphPad, San Diego, CA). The LogIC50 for each experiment was calculated using the former nonlinear regression analysis. Results from four independent experiments were averaged. Significant differences between groups were determined using a 2-tailed unpaired $t$-test.

\section{Results and Discussion:}

\section{Arrestin recruitment by $\mathrm{D} 2-\mathrm{I212} \mathrm{F}$ in response to dopamine}

A BRET assay was utilized to monitor the recruitment of mVenus-arrestin3 to Rluc8tagged D2-WT and D2-I212F. The interaction between D2-WT and arrestin3 is indicated by a dose-dependent increase in the BRET ratio (Fig. $1 A$ ), produced by the activation of the receptor by dopamine. The D2-I212F variant was only $34 \%$ as effective as D2-WT at recruiting arrestin3 (Fig. 1B). 
A

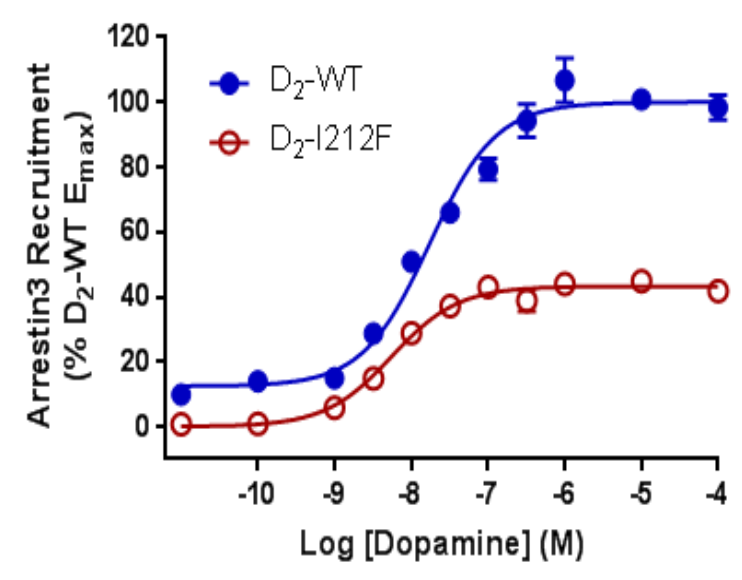

B

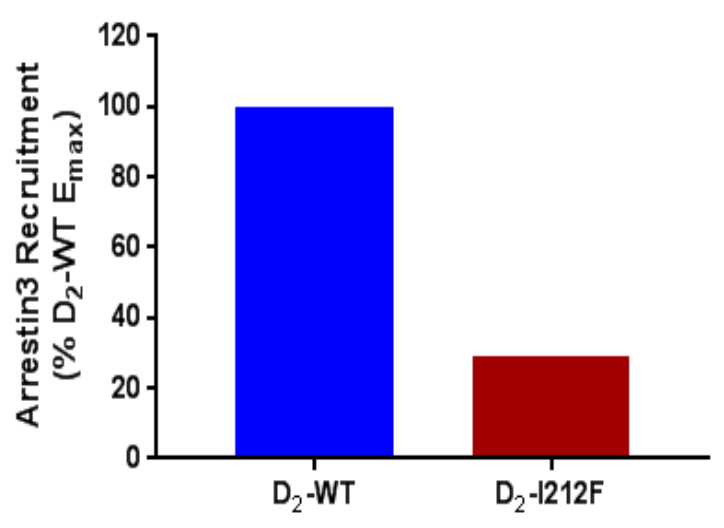

FIGURE 1. D2-mediated arrestin3 recruitment. $A$, D2-WT-Rluc8- and D2-I212F-Rluc8mediated mVenus-Arr3 recruitment in response to incubation with dopamine at the indicated concentrations for $10 \mathrm{~min}$ before measuring receptor/arrestin3 BRET. $B$, maximal mVenusArr3 recruitment by D2-I212F-Rluc8 in response to dopamine stimulation, expressed as a percentage of the maximal activation by $\mathrm{D} 2-\mathrm{WT}$.

Dopamine-induced recruitment of arrestin3 to the receptor was significantly less for the D2I212F variant than for D2-WT, with a $66 \%$ loss of arrestin3 binding. This result agrees with previous findings that demonstrated the necessity of a four-residue segment, residues 212-215 in IL3, for recruitment of arrestin3 (Lan et al., 2009). Lan et al. 2009 demonstrated that the mutation of residues $212-215$ in IL3 resulted in $90 \%$ reduction of the wild type receptor to recruit arrestin. The mutation of residue 212 in IL3 of the D2-I212F variant and the magnitude of its subsequent effect on arrestin3 recruitment, relative to the mutation of residues 212-215, suggests that $\mathrm{I} 212$ alone is important for this function.

\section{D2-I212F-mediated inhibition of cAMP accumulation}

The inhibition of forskolin-induced cAMP accumulation, by the agonist quinpirole, was measured using CAMYEL, a BRET sensor based on the guanine nucleotide exchange factor 
(GEF) Epac. Quinpirole stimulation of D2-WT and D2-I212F produced dose-dependent inhibition of cAMP accumulation; it was observed that lower concentrations of quinpirole were able to fully activate the rare variant, as demonstrated in the quinpirole dose-response curve that is shifted to the left for D2-I212F compared to D2-WT (Fig. 2, $A$ and $B$ ).

A

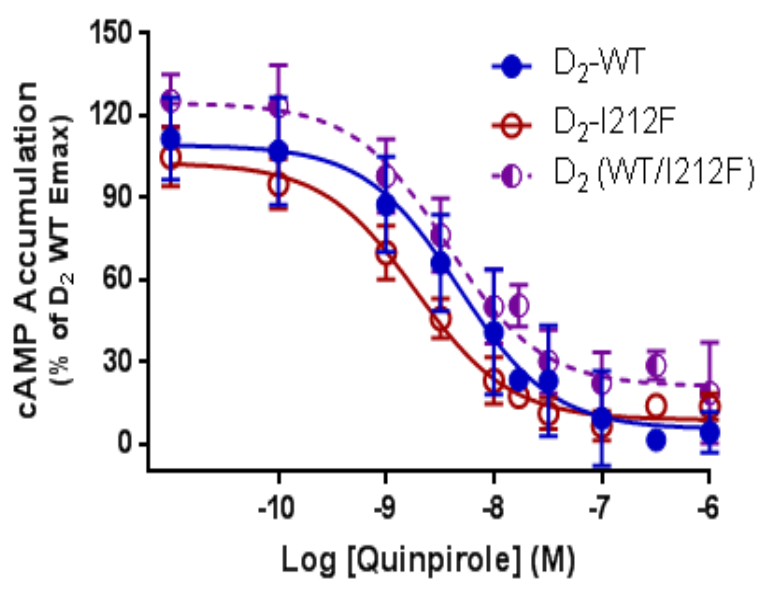

$\mathrm{B}$

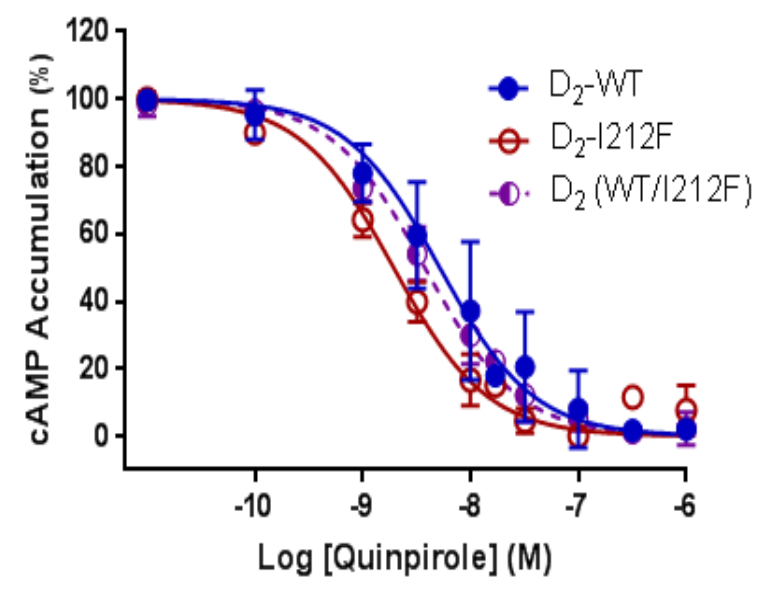

FIGURE 2. D2-mediated inhibition of cAMP accumulation. HEK293 cells were transfected with D2-WT, D2-I212F, or D2(WT/I212F) and CAMYEL, a BRET-based cAMP sensor. The inhibition of forskolin $(10 \mu \mathrm{M})$-stimulated cAMP by quinpirole at the indicated concentrations was measured after $10 \mathrm{~min}$. Dose-response curves are representative of four independent experiments performed with triplicate or quadruplicate samples (mean \pm S.E.). In panel A the results are expressed as a percentage of maximum cAMP production by D2-WT, and in panel B the same results are expressed as a percentage of maximum cAMP production for each condition.

To further investigate whether this missense variant causes movement disorders, a condition that mimics the heterozygosity (D2-WT/D2-I212F), for the SNP affected patients have, was utilized and tested. Co-transfection of D2-WT and D2-I212F trended toward a shift in the EC50, where D2-WT/D2-I212F were different from D2-WT expressed alone, but the difference was not statistically different (Table 1). 


\section{Table 1. Inhibition of cAMP accumulation mediated by D2 receptor in Response to}

Quinpirole The $\operatorname{LogIC}{ }_{50}$ values represent the average of four independent experiments illustrated in Figure 2. $t$-test: $\mathrm{p}<0.05$ for D2-WT vs D2-I212F; $\mathrm{p}<0.05$ for D2-I212F vs D2(WT/I212F); no significant difference was found between D2-WT and D2(WT/I212F).

\begin{tabular}{|c|c|}
\hline & $\begin{array}{c}\text { LogIC }_{\mathbf{5 0}} \\
\text { Mean } \pm \text { SD }\end{array}$ \\
\hline D2-WT & $-8.24 \pm 0.43(\mathrm{n}=4)$ \\
\hline D2-I212F & $-8.72 \pm 0.16(\mathrm{n}=4)$ \\
\hline D2(WT/I212F) & $-8.42 \pm 0.16(\mathrm{n}=5)$ \\
\hline
\end{tabular}

The variant D2-I212F demonstrated its enhanced ability to activate heterotrimeric G proteins, as determined by measuring the inhibition of cAMP accumulation. Additional BRET-based G protein activation assays were performed in the Neve lab, using the same plasmids, and generated results in agreement with the results reported here. Additionally, subsequent experiments in the Neve lab demonstrated that co-transfection of D2-WT and D2-I212F trended toward a shift in the EC50 that was significantly different from D2-WT expressed alone.

\section{Implications and Future Direction:}

The missense variant in D2DR (c.634A>T p.I212F) is likely to be a very rare mutation, but it's important to consider whether the other dozens of $D 2 D R$ SNPs have similar effects on receptor function. Altered D2DR signaling is implicated in brain disorders such as schizophrenia and drug addiction. $D 2 D R$ is one of the most studied markers of susceptibility for schizophrenia; strong associations between schizophrenia and several polymorphisms within $D 2 D R$ have been reported. SNPs in $D 2 D R$ have been shown to influence substance dependence including alcohol and opioid addiction. The results from this study suggest that the missense variant in D2DR 
(c.634A $>$ T p.I212F) is likely to have decreased arrestin-mediated signaling and enhanced G protein-mediated signaling, so behaviors and cellular functions mediated by those two pathways will be altered according to patients with this variant. The next step for this research is to characterize D2-I212F in vivo. The Neve lab is working on producing a mouse model of this genetic disease, which will both confirm that this variant is pathogenic and also make it possible to identify drugs to treat the disorder.

\section{References:}

Beaulieu, J.-M. et al. An Akt/ $\beta$-Arrestin 2/PP2A Signaling Complex Mediates Dopaminergic Neurotransmission and Behavior. Cell 122, 261-273 (2005).

Cepeda, C., Murphy, K., Parent, M. \& Levine, M. The role of dopamine in huntington's disease. Prog. Brain Res. 211, 235-254 (2014).

Clayton, C. C., Donthamsetti, P., Lambert, N. A., Javitch, J. A., \& Neve, K. A. Mutation of three residues in the third intracellular loop of the dopamine D2 receptor creates an internalization-defective receptor. The Journal of Biological Chemistry 289, 3366333675 (2014).

DeWire, S. M., Ahn, S., Lefkowitz, R. J. \& Shenoy, S. K. $\beta$-Arrestins and Cell Signaling. Annual Review of Physiology 69, 483-510 (2007).

Donthamsetti, P., Quejada, J. R., Javitch, J. A., Gurevich, V. V. \& Lambert, N. A. Using Bioluminescence Resonance Energy Transfer (BRET) to Characterize Agonist-Induced Arrestin Recruitment to Modified and Unmodified G Protein-Coupled Receptors. Current protocols in pharmacology 70, 2.14 .1 (2015).

Hilger, D., Masureel, M. \& Kobilka, B. K. Structure and dynamics of GPCR signaling complexes. Nature Structural \& Molecular Biology 25, 4-12 (2018). 
Jiang, L. I. et al. Use of a cAMP BRET Sensor to Characterize a Novel Regulation of cAMP by the Sphingosine 1-Phosphate/G 13 Pathway. Journal of Biological Chemistry 282, 1057610584 (2007).

Lan, H., Liu, Y., Bell, M. I., Gurevich, V. V. \& Neve, K. A. A dopamine D2 receptor mutant capable of $\mathrm{G}$ protein-mediated signaling but deficient in arrestin binding. Molecular pharmacology 75, 113 (2009).

Liu, Y., Buck, D. C., Macey, T. A., Lan, H. \& Neve, K. A. Evidence That Calmodulin Binding to the Dopamine D 2 Receptor Enhances Receptor Signaling. Journal of Receptors and Signal Transduction, 2007, Vol.27(1), p.47-65 27, 47-65 (2007).

Lizarraga, K. J., Gorgulho, A., Chen, W. \& De Salles, A. A. Molecular imaging of movement disorders. World journal of radiology 8, 226 (2016).

Matthiesen, K. \& Nielsen, J. Cyclic AMP Control Measured in Two Compartments in HEK293 Cells: Phosphodiesterase KM Is More Important than Phosphodiesterase Localization. PLOS ONE 6, e24392 (2011).

Neve, K. A. (ed.) (2010). The Dopamine Receptors, New York, NY: Humana Press.

Pierce, K. L. \& Lefkowitz, R. J. Classical and new roles of $\beta$-arrestins in the regulation of GPROTEIN-COUPLED receptors. Nature Reviews Neuroscience 2, 727 (2001).

Pfleger, K. D. G., Seeber, R. M. \& Eidne, K. A. Bioluminescence resonance energy transfer (BRET) for the real-time detection of protein-protein interactions. Nature Protocols $\mathbf{1}$, 337-345 (2006).

Salahpour, A. BRET biosensors to study GPCR biology, pharmacology, and signal transduction. Frontiers in Endocrinology 3, (2012). 
Stoddart, L. A. et al. Application of BRET to monitor ligand binding to GPCRs. Nature Methods 12, 661-663 (2015).

Tuteja, N. Signaling through G protein coupled receptors. Plant signaling \& behavior 4, 942947 (2009).

Usiello, A. et al. Distinct functions of the two isoforms of dopamine D2 receptors. Journal of Experimental Psychology: Human Perception and Performance 408, 199-203 (2000).

Valkovic, A. L. et al. Real-time examination of cAMP activity at relaxin family peptide receptors using a BRET-based biosensor. Pharmacology Research \& Perspectives 6 , e00432 (2018)

Xu, Y., Piston, D. W. \& Johnson, C. H. A bioluminescence resonance energy transfer (BRET) system: application to interacting circadian clock proteins. Proceedings of the National Academy of Sciences 96, 151-156 (1999). 